Table 1. The distribution of US-GLOESS according to different categories by indices

\begin{tabular}{|c|c|c|c|c|}
\hline & $\begin{array}{c}\text { GLOESS } \\
38 \text { joints } \\
\text { mean } \pm S D\end{array}$ & $\begin{array}{c}\text { GLOESS } \\
28 \text { joints } \\
\text { mean } \pm S D\end{array}$ & $\begin{array}{c}\text { Joint counts } \\
\geq \text { score } 2 \\
\text { mean } \pm S D\end{array}$ & $\begin{array}{c}\text { Joint counts } \\
\text { with score } 3 \\
\text { mean } \pm S D\end{array}$ \\
\hline \multicolumn{5}{|l|}{ DAS-28 } \\
\hline Remission ( $\mathrm{n}=77$ ) & $10.9 \pm 7.5$ & $7.8 \pm 7.2$ & $3.5 \pm 3.1$ & $0.7 \pm 1.3$ \\
\hline $\operatorname{LDA}(n=11)$ & $14.5 \pm 6.6$ & $10.0 \pm 5.4$ & $4.9 \pm 3.0$ & $0.9 \pm 1.4$ \\
\hline $\operatorname{VMDA}(n=5)$ & $9.6 \pm 6.2$ & $5.4 \pm 3.5$ & $3.0 \pm 2.3$ & $0.80 \pm 1.7$ \\
\hline \multicolumn{5}{|l|}{ CDAI } \\
\hline Remission $(\mathrm{n}=48)$ & $10.0 \pm 6.9$ & $6.9 \pm 6.2$ & $3.02 \pm 2.7$ & $0.60 \pm 1.0$ \\
\hline LDA $(n=48)$ & $12.7 \pm 7.7$ & $9.1 \pm 7.2$ & $4.3 \pm 3.3$ & $0.94 \pm 1.5$ \\
\hline \multicolumn{5}{|l|}{ SDAI } \\
\hline Remission ( $n=42)$ & $9.9 \pm 6.6$ & $6.6 \pm 5.9$ & $2.9 \pm 2.6$ & $0.5 \pm 0.9$ \\
\hline $\operatorname{LDA}(n=50)$ & $12.4 \pm 7.8$ & $9.1 \pm 7.5$ & $4.2 \pm 3.3$ & $0.9 \pm 1.5$ \\
\hline $\operatorname{MDA}(n=1)$ & 13.0 & 4.0 & 5.0 & 0 \\
\hline \multicolumn{5}{|l|}{ RAPID } \\
\hline Remission $(n=36)$ & $10.3 \pm 6.2$ & $6.7 \pm 4.6$ & $3.1 \pm 2.6$ & $0.5 \pm 0.8$ \\
\hline Low activity $(n=28)$ & $9.6 \pm 5.8$ & $6.6 \pm 4.6$ & $2.7 \pm 2.2$ & $0.3 \pm 0.7$ \\
\hline MDA $(n=30)$ & $14.0 \pm 9.4$ & $10.7 \pm 9.7$ & $5.1 \pm 3.9$ & $1.4 \pm 1.9$ \\
\hline $\operatorname{HDA}(n=2)$ & $15 \pm 1.4$ & $8.5 \pm 2.1$ & $5 \pm 1.4$ & 0 \\
\hline
\end{tabular}

Conclusions: Our results show that CDAI is superior then other clinical indices to assess remission in RA. US has the superiority over clinical indices to predict flares and 28 joint GLOESS is superior to 38 joints.

Disclosure of Interest: None declared

DOI: 10.1136/annrheumdis-2017-eular.3227

\section{SAT0064 HIGH MMP3 SERUM LEVELS ARE ASSOCIATED WITH EXTENSIVE STRUCTURAL DAMAGE IN PATIENTS WITH EARLY, TREATMENT NAIVE RHEUMATOID ARTHRITIS (RA): TWO YEARS PROSPECTIVE CLINICAL AND ULTRASONOGRAPHIC STUDY}

S.Z. Prodanovic ${ }^{1}$, G. Radunovic ${ }^{2}$, M. Sefik-Bukilica ${ }^{3}$, M. Zlatanovic ${ }^{4}$,

K. Simic-Pasalic ${ }^{5}$, S. Seric 6 , N. Damjanov ${ }^{7} .{ }^{1}$ Clinical IVb; ${ }^{2}$ Deputy of head;

${ }^{3}$ Clinical IVa; ${ }^{4}$ Clinical III; ${ }^{5}$ Clinical VI; ${ }^{6}$ Radiology Department; ${ }^{7}$ Head of Institute, Institute of Rheumatology, Belgrade, Serbia

Objectives: To investigate the association of high baseline MMP-3 serum levels with hands and feet joints structural damage progression, estimated by ultrasonography (US), in patients with early, treatment "naïve" RA without X-ray visible erosions.

Methods: Sixty-three pts. (9 males and 54 females; mean age 53.4 yrs 21$81 \pm 14.1$ ) with early RA (EULAR/ACR 2010 criteria) and symptom duration of $\leq 12$ months (mean duration of 3.8 months) had baseline serum MMP-3 levels tested. Patients had been DMARDs/glucocorticoid naïve, without visible X-ray erosions at the study entry. The subsequent structural joints damages, that were estimated by high frequency linear probe by ESAOTE My Lab 70 machine, as well as clinical markers of disease activity, in the first 2 years were followed. The presence of bone erosion was analyzed at the wrist, MCP2 and MCP5 joints of both hands, as well as at MTP5 joints according to OMERACT US group definition. In order to estimate progression of preexisting erosion the total volume (TV) of bone erosion were calculated by multiplying three diameters $(\mathrm{mm})$ : a-the length of erosion; diameter $b$ - the width and diameter $c$-the depth of erosion. Anova statistical method was performed in data processing.

Results: 46 pts. had basal serum MMP-3 level higher than normal (MMP-3 positive). The 504 joints were assessed summary by US on each visit. The 122 bone erosions in total (1.9 per patient) were depicted at baseline and 213 bone erosions (4.3 per patient) at follow-up visit in whole group. After 24 months MMP3 positive pts. had significantly higher total number of US erosions than MMP-3 negative (3.8 vs. 2.4, $\mathrm{p}=0.039$ ). The total volume of bone erosion $\left(\mathrm{mm}^{3}\right)$ was higher in MMP-3 positive than MMP-3 negative pts. after 24 months of treatment, but without statistical significance (18.6 vs. 8.9, $p=0.07$ ). MMP-3 positive pts. had a significantly higher value of ESR, CRP and DAS28 than MMP-3 negative pts. at the baseline visit (53.6 vs. $25.9, p=0.002 ; 36.6$ vs. $9.5, p=0.005 ; 6.0$ vs. $4.8, p=0.002$, respectively). All of those parameters were significantly decreased after 24 months in a group of MMP-3 positive compared to MMP-3 negative pts. $(p=0.009, p=0.021, p=0.028$, respectively).

Conclusions: After 2-year of follow-up, US assessment showed a significantly higher number of new bone erosions in patients with early, treatment "naïve" RA and baseline MMP3 levels higher than normal (MMP3 positive) compared to patients with normal baseline levels of MMP3, as well as a biger TV of bone erosions but not statistically significant. The parameters of RA activity (ESR, CRP, DAS28) significantly correlated with baseline MMP-3 levels of higher than normal. Disclosure of Interest: None declared

DOI: 10.1136/annrheumdis-2017-eular.4002

\section{SAT0065 ACPA AGAINST DIFFERENT CITRULLINATED PEPTIDES IDENTIFY SPECIFIC PHENOTYPES OF RHEUMATOID ARTHRITIS}

M. Brink ${ }^{1}$, M. Hansson ${ }^{2}$, L. Mathson-Alm ${ }^{3}$, M. Cornillet ${ }^{4}$, J. Rönnelid ${ }^{3}$, K. Skriner ${ }^{5}$, G. Serre ${ }^{4}$, R. Holmdahl ${ }^{6}$, L. Klareskog ${ }^{2}$, S. Rantapää-Dahlqvist ${ }^{1}$

${ }^{1}$ Public Health and Clinical Medicine, Rheumatology, Umeå University, Umeå;

${ }^{2}$ Rheumatology Unit, Dept of Medicine, Karolinska Institute, Stockholm;

${ }^{3}$ Immunology, Genetics and Pathology, Uppsala University, Uppsala, Sweden; ${ }^{4} U$ 1056 Inserm, Univeristy de Toulouse, Toulouse, France; ${ }^{5}$ Medicine, Charité University, Berlin, Germany; ${ }^{6}$ Medical Inflammation Research, Karolinska Institute, Stockholm, Sweden

Background: Anti-citrullinated protein/peptide antibodies (ACPA) have been suggested to identify a more severe phenotype of rheumatoid arthritis (RA).

Objectives: In this study in an inception cohort of early RA we have analysed a number of antibodies against different citrullinated and/or mutated peptides using a multiplex platform in relation to the patients disease inflammation and radiological destruction

Methods: Patients with early RA ( $\leq 12 \mathrm{~m}$ of symptoms) fulfilling the 1987 ARA criteria ( $n=1022,692 \mathrm{f} / 330 \mathrm{~m}$, mean age $56.7 \pm 14.0$ years) were sampled at the time of diagnosis and assessed using disease activity score (DAS28) at baseline, $6,12,18$ and 24 months. Radiographs were graded using Larsen score (baseline and at $24 \mathrm{~m}$ ). Plasma sampled at baseline was analysed for presence of antibody reactivities against 21 different citrullinated peptides/proteins; Fibrinogen (Fib) $\alpha 36-50$, Fib $\alpha 573$, Fib $\alpha 591$, Fib $\alpha 621-635$, Fib $\beta 36-52$, Fib $\beta 60-74$, Fib $\beta 62-78$ (72), Fib362-78 (74), Filaggrin (Fil307-324), $\alpha$-Enolase peptide 5-21 (CEP-1), Vimentin (Vim) 2-17, Vim60-75, F4-R-Cit, F4-Cit-Cit, F4- Cit-R), or mutated proteins (Bla26, Pept1, Pept5, PeptZ1, PeptZ2) and type II Collagen citrullinated or not using a custom-made microarray assay based on the ImmunoCAP ISAC system (Phadia AB, Sweden). Cut-off levels were at the 98th percentile of controls $(n=477)$. Anti-CCP2 was analysed using ELISA (Euro Diagnostica, Sweden).

Results: The most frequent appearing ACPA were; Fib $\beta 60-74$ (63\%), Vim60-75 (56.6\%), FibB36-52 (55.1\%), Fil307-324 (54.9\%), CEP-1 (53.7\%) and Pept5 $(52.0 \%)$ besides CCP2 (67.5\%). Adding all ACPAs gave additional $13.1 \%$ of positivity in the anti-CCP2 negative group, yielding a positivity of $77.5 \%$. The median (IQR) number of positive ACPA-peptide was 8 (11). There was a high degree of correlation between the antibodies, e.g., anti-Fib $\beta 60-74$ vs. -Vim60-75, -Fib336-52 or anti-CCP2 antibodies and also anti-Fil307-324 vs. -FibB36-52, -F4 R-Cit or anti-CCP2 antibodies (rs 0.692-0.79). Positivity for all antibodies was associated with higher ESR (baseline and $\mathrm{AUC}_{24}$ ). A number of antibodies were associated with both high DAS28 (baseline and $\mathrm{AUC}_{24}$ ) and radiological findings/progression (anti-CCP2, - Fil307-324, -Vim60-75 and Vim 2-17, and -CEP1 antibodies), whilst some others were more associated with inflammation (DAS28, baseline and $\mathrm{AUC}_{24}$ ) (anti-FibB60-74, -Pept5 and -F4R-cit antibodies) and others more with radiological destruction/progression (anti-Fib/336-52, Fib $\beta 74$, -PeptZ1, -F4 Cit-R antibodies). Partial least squares regression analyses confirmed the results with significant correlation between radiological progression and antibodies against Vim2-17, Fib336-52, CEP1, Fiba621-635, and CCP2 and between DAS28AUC 24 and Vim60-75, Vim2-17, Fib $\alpha 621-635$ and F4R-Cit. Patients treated with biologics during the first 24 months $(11.2 \%)$ were significantly more frequent positive for anti-CCP2, -Vim60-75, -Fib $\alpha 36-50$, -PeptZ1 and -PeptZ2 antibodies vs. being negative.

Conclusions: Analyses at baseline, of the ACPA specificity profiles allowed different patterns of disease activity and radiological progression during the first 24 months of the disease to be identified.

Disclosure of Interest: None declared

DOI: 10.1136/annrheumdis-2017-eular.5085

\section{SAT0066 HISTOLOGICAL AND ULTRASOUND SYNOVIAL PREDICTORS OF CLINICAL DIFFERENTIATION TO DEFINED ARTHRITIS IN PATIENTS WITH SERONEGATIVE UNDIFFERENTIATED PERIPHERAL INFLAMMATORY ARTHRITIS}

S. Alivernini ${ }^{1}$, L. Petricca ${ }^{1}$, B. Tolusso ${ }^{1}$, L. Bui ${ }^{2}$, C. Di Mario ${ }^{1}$, M.R. Gigante ${ }^{1}$ G. Di Sante ${ }^{1}$, R. Benvenuto ${ }^{2}$, A.L. Fedele ${ }^{1}$, F. Federico ${ }^{2}$, E. Gremese ${ }^{1}$,

G. Ferraccioli ${ }^{1}$. ${ }^{1}$ Institute of Rheumatology; ${ }^{2}$ Institute of Pathology, Catholic University of the Sacred Heart, Rome, Italy

Background: Undifferentiated Peripheral Inflammatory Arthritis (UPIA) is a common diagnosis at the first clinical evaluation in rheumatological settings. However, the likelihood of developing a well-defined rheumatic disease in UPIA patients is still matter of debate.

Objectives: To examine the role of ultrasound (US) and histological parameters in the disease outcome of patients with seronegative UPIA.

Methods: Fourty-two patients with $\lg \mathrm{A} / \mathrm{lgM}$-Rheumatoid Factor and anticitrullinated peptide antibodies negative UPIA, naïve to any Disease-Modifying Anti-Rheumatic Drugs, underwent Gray Scale (GSUS) and power Doppler (PDUS) evaluation and US guided synovial tissue biopsy. Synovial expression of CD68, CD3, CD21, CD20 and CD31 was evaluated by immunohistochemistry. IL-6, VEGF-A and VEGF-D peripheral blood (PB) and synovial fluid (SF) levels were measured by ELISA. To exclude Reactive Arthritis, each patient underwent genital and throat swabs. Afterwards, each UPIA patient was treated with chloroquine $250 \mathrm{mg} /$ daily and followed every 3 months for 1 year and classified as having 
UPIA, Rheumatoid Arthritis (RA), Spondyloarthritis $(\mathrm{SpA})$ or Psoriatic Arthritis (PsA), respectively.

Results: During the follow-up 6 (14.3\%) UPIA reached a defined diagnosis (2 RA, $2 \mathrm{SpA}$ and 2 PsA, respectively). At baseline, UPIA who differentiated had higher GSUS $(p=0.01)$ and PDUS scores $(p=0.02)$ compared to patients who remained as UPIA within 1 year. At baseline, UPIA who differentiated towards defined arthritis had higher histological scores for lining and sublining $\operatorname{CD}^{+} 8^{+}(p=0.005$ and $p=0.04$ for lining and sublining, respectively), sublining $C D 3^{+}$cells $(p=0.002)$ and $C D 31^{+}$vessels count $(p<0.001)$ than patients who remained as UPIA. In addition, there were direct correlations between baseline GSUS and PDUS scores with lining $C D 68^{+}$cells scores $(p<0.001$ for GSUS and $p=0.02$ for PDUS scores respectively), sublining $C D 68^{+}$cells scores ( $p=0.02$ for GSUS and $p=0.03$ for PDUS scores respectively), sublining $\mathrm{CD}^{+}$cells score $(\mathrm{p}=0.002$ for GSUS and $p=0.002$ for PDUS scores respectively) and CD $31^{+}$vessels count $(p<0.001$ for GSUS and $\mathrm{p}=0.01$ for PDUS scores respectively) in UPIA. Finally, the areas under the receiver operating characteristic (ROC) curves $C D 31^{+}$vessels count (cut-off value: 24.3), GS score (cut-off value: 1.5) and PDUS score (cut-off value: 1.5) were calculated to assess the best cut-off points to identify the differentiation likelihood during the follow-up in UPIA patients. The logistic regression analysis, demonstrated that having baseline GSUS and PDUS scores $\geq 1.5$ [OR:13.64 (95\% Cl: 0.98-242.59); $\mathrm{p}=0.05]$ and $\mathrm{CD} 31^{+}$vessels count $\geq 24.3$ [OR:51.13 (95\% Cl: 3.15-829.16); $p=0.01]$ were independent factors associated with the achievement of defined arthritis.

Conclusions: Histological and US assessment may help in the identification of patients with seronegative UPIA with high likelihood of clinical differentiation towards defined arthritis.

Disclosure of Interest: None declared

DOI: 10.1136/annrheumdis-2017-eular.4841

\section{SAT0067 FURTHER TREATMENT INTENSIFICATIONS IN UNDIFFERENTIATED AND RHEUMATOID ARTHRITIS PATIENTS ALREADY IN LOW DISEASE ACTIVITY HAVE LIMITED BENEFIT TOWARDS PHYSICAL FUNCTIONING}

S.A. Bergstra ${ }^{1}$, O. Olivas ${ }^{2}$, G. Akdemir ${ }^{1}$, N. Riyazi ${ }^{3}$, G. Collee ${ }^{3}$, J.H. Van Groenendael $^{4}$, R.B. Landewé ${ }^{5,6}$, C.F. Allaart ${ }^{1} .{ }^{1}$ Rheumatology, LUMC, Leiden, Netherlands; ${ }^{2}$ Rheumatology, Hospital Universitario 12 de Octubre, Madrid, Spain; ${ }^{3}$ Medical Center Haaglanden, The Hague; ${ }^{4}$ Reumazorg Southwest Netherlands, Bergen op Zoom; ${ }^{5}$ Amsterdam Rheumatology \& Immunology Center, Amsterdam; ${ }^{6}$ Zuyderland Medical Center, Heerlen, Netherlands

Background: It is recommended to optimize treatment as long as a predefined treatment target is not met, but should we aim at remission if patients are in low disease activity (LDA)?

Objectives: To assess if RA or undifferentiated arthritis (UA) patients who achieved LDA benefit with better functional ability from treatment intensification aimed at DAS remission.

Methods: In the IMPROVED study 610 patients with early RA (ACR 2010) or UA were "treated to target" aimed at DAS remission, assessed 4-monthly. Initial treatment was methotrexate (MTX) + tapered high dose prednisone. Patients with DAS $\leq 1.6$ tapered treatment. Patients with DAS $>1.6$ were randomized to MTX + hydroxychloroquine + sulphasalazine + prednisone or to MTX + adalimumab. Over 5 years, patients with DAS $>1.6$ were required to increase, change or restart medication. HAQ was measured 4-monthly. A linear mixed model analysis with random intercept was performed to test the relationship between changes in therapy and HAQ over time. Patients in LDA with DAS $>1.6$ with and without (i.e. protocol violation) treatment change were compared. $\triangle \mathrm{HAQ}$ and $\triangle \mathrm{DAS}$ at each visit compared to the previous visit were calculated. We tested the interaction effect between change in treatment and follow-up time adjusted for possible confounders.

Results: Overall, over 5 years DAS (baseline mean (SD) 3.2 (1.7)) and HAQ (1.2 (0.7)) showed a statistically significant and clinically relevant decrease $(\triangle \mathrm{HAQ}$ $-0.59,95 \% \mathrm{Cl}-0.61,-0.57 ; \Delta \mathrm{DAS}-1.77,95 \% \mathrm{Cl}-1.79 ;-1.75)$. The number of patients in LDA per visit ranged from 88 to 146 , of which $26 \%$ to $73 \%$ (increasing over time) had no treatment change due to protocol violations. We found a statistically significant but not clinically relevant effect of treatment change on $\triangle \mathrm{HAQ}$, corrected for baseline $\mathrm{HAQ}$, age, gender and treatment arm (model 1 , $\beta-0.085,95 \% \mathrm{Cl}-0.13,-0.044$ ). When $\triangle \mathrm{DAS}$ was added (model 2), the effect of treatment change was partly explained by $\triangle \mathrm{DAS}$ and no longer statistically significant $(\beta-0.022,95 \% \mathrm{Cl}-0.060 ; 0.015)$. The effect of treatment intensification on HAQ improvement became less over time, as demonstrated by a statistically significant interaction between change in HAQ and time in follow-up in model 3 ( $\beta$ $0.0098,95 \% \mathrm{Cl} 0.0010 ; 0.019$ ) (table 1).

Conclusions: Treatment intensification in early RA or UA patients who have already achieved low disease activity is associated with a statistically significant decrease in HAQ, but not with a clinically meaningful improvement in functional ability. The effect on $\triangle \mathrm{HAQ}$ decreased with increasing follow-up time. Therefore not remission or low disease activity, but good functional ability may be the optimal treatment target at which to steer treatment adjustments. These results suggest that, whereas remission may be the optimal goal, when patients in low disease activity have acceptably low $\mathrm{HAQ}$, further treatment intensification may only have downsides such as side effects and costs.

Disclosure of Interest: None declared
Table 1: Linear Mixed Model analysis to assess the effect of treatment intensification on change in $\mathrm{HAQ}$.

\begin{tabular}{|c|c|c|c|}
\hline & $\beta$ & $\mathbf{P}$ & $95 \% \mathrm{~d}$ \\
\hline \multicolumn{4}{|l|}{ Model $1(n \text { patients }=479, n \text { visits }=1528)^{\circ}$} \\
\hline Treatment intensification & -0.085 & $<0 . \infty 1$ & $-0.13 ;-0.044$ \\
\hline Follow-up time & 0.0057 & 0.019 & $0.00094 ; 0.010$ \\
\hline \multicolumn{4}{|l|}{ Model 2 ( $n$ patients $=476, n$ visits $=1509)^{*}$} \\
\hline Treatment intensification & -0.022 & 0.246 & $-0.060 ; 0.016$ \\
\hline Follow-up time $e^{b}$ & 0.0022 & 0.313 & $-0.0021 ; 0.0066$ \\
\hline DAS change & 0.23 & $<0 . \infty 1$ & $0.21 ; 0.26$ \\
\hline \multicolumn{4}{|l|}{ Model $3(n \text { patients }=476, n \text { visits }=1509)^{\circ}$} \\
\hline Treatment intensification & -0.10 & 0.013 & $-0.18 ;-0.021$ \\
\hline Follow-up time & -0.0034 & 0.323 & $-0.010 ; 0.0033$ \\
\hline Treatment intensification " follow-up time & 0.0098 & 0.029 & $0.0010 ; 0.019$ \\
\hline DAS change & 0.23 & $<0.001$ & $0.21 ; 0.26$ \\
\hline
\end{tabular}

$\mathrm{HAQ}=$ health assessment questionnaire, $\mathrm{SE}=$ standard error, $\mathrm{Cl}=$ confidence interval.

-All models were adjusted for age, gender, treatment arm and baseline HAQ.

"Follow-up time is added to the model as visit number, with time between visits being

4 months

DOI: 10.1136/annrheumdis-2017-eular.1606

\section{SAT0068 THE TIME UNTIL PERFORMING TIGHT CONTROL AS A TREAT-TO-TARGET STRATEGY AND THE TOLERABILITY OF METHOTREXATE STRONGLY INFLUENCE THE ACHIEVEMENT OF CLINICAL REMISSION IN RHEUMATOID ARTHRITIS}

T. Hagiwara, K. Kamada, N. Namura. Rheumatology, Takarazuka City Hospital, Takarazuka, Japan

Background: Clinical remission (CR) is the first targeted outcome of early treatment for rheumatoid arthritis (RA). Therefore, a consensus is needed for achieving CR by using the treat-to-target (T2T) strategy in RA treatment. However, in patients who received long-term insufficient treatment for RA, achievement of CR becomes increasingly difficult, especially if there is delay in the treatment.

Objectives: We aimed to examine factors that hinder successful RA treatment. We believe that making primary-care physicians aware of treatment results will increase the remission rate of RA.

Methods: We examined 388 patients with RA who were observed between January and October 2016 and who had not received new disease-modifying anti-rheumatic drugs (DMARDs) more than 3 months before the observation day. We investigated their age at RA onset, sex, Steinbrocker radiographic stage and functional class, activity level, rheumatoid factor (RF), the anti-cyclic citrullinated peptide antibody and DMARDs prescribed at the first consultation (Prescribed Before), disease activity, status of methotrexate (MTX), glucocorticoids (GCs), and biologic agent use at the last observational day.

First, we analysed the assumed remissions by using the Boolean-based definition (Boolean remission) as a purpose variable for these factors. Furthermore, we examined the odds ratio (OR) and $95 \%$ confidence interval $(95 \% \mathrm{Cl}$ ) by using a multiple logistic regression analysis for the statistically significantly different factors.

As for the time-related factor, we recognized that each factor had distinct multiplex collinear characteristics. Therefore, we adopted the time required for the first consultation as disease duration with the most effective values as the analysis object. The representative factor for the functional assessment adopted class according to the number of effective analyses.

Results: We recognised the statistically significant differences in disease duration, stage, class at the time of the first medical examination, RF, Prescribed Before, and state of MTX and GCs use at the last observation day for the achievement of Boolean remission.

We examined the multiple logistic regression analysis with the previously mentioned results and obtained the following results.

- Disease duration (per 1 year); OR 1.110, 95\% Cl 1.048-1.175, $p<0.001$.

- MTX (state; using vs no using); OR 2.522, 95\% Cl 1.560-4.076, p $<0.001$

- Class at 1st interview; OR 1.512, 95\% Cl 1.126-2.029, $\mathrm{p}<0.01$

- GCs (state; no using vs using); OR 1.803, 95\% Cl 0.912-3.565, p=0.090.

- Disease duration ( $<1.605 y$ vs $>1.605 y)$; OR $2.233,95 \% \mathrm{Cl} 1.437-3.470$, $\mathrm{p}<0.001$.

- MTX (state; using vs no using); OR 2.656, 95\% CI 1.644-4.291, p $<0.001$

- Class at 1st interview; OR 1.589, 95\% Cl 1.181-2.136, $\mathrm{p}<0.01$.

- GCs (state; no using vs using); OR 1.883, 95\% Cl 0.956-3.711, p=0.067.

Conclusions: Our results indicated the importance of the time required for consultation facilities with the T2T strategy treatment, tolerability for MTX use, and mild dysfunction at the first interview.

The window of opportunity to achieve remission for patients with RA has less time than expected. Therefore, we recommend that physicians should introduce patients with RA to a rheumatologist following the T2T strategy promptly when the primary care provided by the family physician is insufficient. 